charismatischen Führer[s]« Chruschtschow konnte Ritvo zufolge die Sowjetunion zum damaligen Zeitpunkt weiterhin als ein »totalitäres System ${ }^{597}$ charakterisiert werden. ${ }^{598}$

\title{
2.9 Analytisches Resümee
}

Auch wenn der Der Monat keine Zeitschrift war, in der wissenschaftliche Forschung im unmittelbaren Sinne betrieben wurde, kann allerdings mit Blick auf die Thematisierung, Beschreibung und Analyse der Geschichte des sowjetischen Kommunismus seit der Oktoberrevolution von 1917 bis 1960 konstatiert werden, dass unter den $\mathrm{Au}$ toren, zumal den zahlreichen Renegaten, eine Diskussion über eben dieses komplexe Phänomen in Gang kam, die neben einem politischen und theoretischen auch einen wissenschaftlichen Charakter besaß. Die von den 122 Autoren geschriebenen knapp 250 Beiträge zum Thema der facettenreichen Geschichte des (internationalen) Sowjetkommunismus stellten zweifelsohne auch den Versuch dar, auf der Grundlage der zeitgenössischen Forschungsliteratur eine genuine Analyse der Konstituierung und Entwicklung des sowjetkommunistischen Herrschaftssystems zu erstellen - freilich in Anbetracht von fehlenden elementaren schriftlichen »Dokumenten und Statistiken« respektive der angesprochenen grundsätzlichen »Archivsituation«.

Vor dem Hintergrund der Auseinandersetzung mit der Vorgeschichte, d.h. den näheren historisch-politischen und -gesellschaftlichen sowie ideengeschichtlichen Bedingungen in Russland des 19. und zu Beginn des 20. Jahrhunderts, wurde im Monat ausdrücklich darauf eingegangen, wie es der bolschewistischen Partei unter der Führung Lenins im Oktober 1917 als »Minderheit« gelang, die politische Macht zu ergreifen und sie mit »allen« Mitteln zu verteidigen. Vor allem wurde hierbei hervorgehoben, dass die Machtergreifung der Bolschewiki keineswegs zwangsläufig erfolgte. Denn: Ohne zum Beispiel den Ausbruch des Ersten Weltkrieges und die sich vor allem durch den Verlauf des Krieges extrem zugespitzte soziale, wirtschaftliche und politische Situation im Land wären die Bolschewiki auch in naher Zukunft aller Voraussicht nach eine politische »Sekte« geblieben. Wenn zudem die demokratisch legitimierte »Kerenski-Regierung« im Oktober 1917 die Entscheidung getroffen hätte, mit Waffengewalt die staatliche Macht gegen die »nur« einige tausend Mann starken revolutionären Bolschewiken zu verteidigen, wäre es möglicherweise nicht zur Konstituierung des kommunistischen Einparteienregimes, der »Diktatur des Proletariats«, und mithin zur Abschaffung der zum damaligen Zeitpunkt gewonnenen - wenn auch nur rudimentär vorhandenen - »rechtsstaatlich« gesicherten bürgerlichen demokratischen Grundrechte gekommen. In diesem Zusammenhang kam im Monat sehr genau zum Ausdruck, dass die Bolschewiki nach der staatlichen Machteroberung zur Sicherung

597 Ebd., S. 13.

598 Vgl. in diesem Zusammenhang auch die grundsätzlichen, im Hinblick auf den in der vorliegenden Arbeit insgesamt zugrunde liegenden Untersuchungszeitraum, der bekanntlich mit dem Heft 139 im Jahre 1960 endet, sozusagen abschließenden Einschätzungen und Reflexionen zur Ceschichte der Sowjetunion nach der Revolution von 1917 von George f. Kennan, die sich auch mit der möglichen zukünftigen Entwicklung des sowjetischen Regimes unter den Bedingungen einer »modernen Industriegesellschaft« beschäftigten, in: Melvin ]. Lasky, Ein Gespräch mit Georgef. Kennan, in: Der Monat 12 (1960), H. 136, S. 5-15, hier bes. S. $13 \mathrm{ff}$. 
ihrer Herrschaft mit der im Dezember 1917 in Petrograd ins Leben gerufenen "Außerordentlichen Kommission zur Bekämpfung von Konterrevolution", der Tscheka (später GPU/NKWD/KGB) unter der Leitung von Feliks Dzierzynski einen Geheimdienstapparat schufen, mit dem einerseits dem »weißen Terror « der »rote Terror « entgegengesetzt werden sollte, der andererseits allerdings alsbald zu einem Instrument wurde, um den Terror gegen sämtliche »objektiven Feinde« der Revolution zu richten. Denn nach dem siegreichen Ende im auf beiden Seiten mit extremer Gewalt geführten Bürgerkrieg Anfang 1921 wurde die Tscheka zu einem schnell wachsenden Herrschaftsinstrument des neuen Regimes, das den Terror keineswegs ausschließlich gegen den ideologischpolitischen »Klassenfeind « einsetzte, sondern auch gegen Menschewiki, Anarchisten, Sozialrevolutionäre und - wie bei der blutigen Unterdrückung der sozialen und politischen Proteste im Frühjahr 1921 in Petrograd und Kronstadt - gegen die eigene Bevölkerung. Vor dem Hintergrund der Thematisierung der blutigen »Säuberungswelle« gegen die »konterrevolutionären Elemente« mit »nächtlichen Massenerschießungen « wurde im Monat zudem auf das Verhaften und Verschleppen von zahlreichen Aufständischen durch Tschekaeinheiten in die sibirischen Gefängnisse und Konzentrationslager ausdrücklich eingegangen. Bei der Fokussierung auf den frühen Terror des sowjetischen Regimes in den ersten Jahren nach der Machteroberung wurde auch darauf hingewiesen, dass bereits unter Lenin im Jahre 1922 gegen führende Vertreter der Sozialrevolutionären Partei ein erster sgroßer Moskauer Prozess stattfand, der bereits das Vorspiel für die in den 1930er-Jahren durchgeführten Schauprozesse darstellte, und dass die Anfänge des Systems der Konzentrationslager bereits im Leninismus lagen. Mit anderen Worten: Im Monat wurde der Fokus bei der Beschreibung und Analyse des Verlaufs der Russischen Revolution auch auf die historische Tatsache gelegt, dass bei allen (qualitativen und quantitativen) politischen, ideologischen und auch individuellen Differenzen zwischen dem Terror unter Lenin und später unter Stalin keine absolute Trennungslinie gezogen werden konnte.

Bei der Beschreibung und Analyse des stalinistischen Herrschaftssystems lag in den zahlreichen Veröffentlichungen im Monat der eigentliche Schwerpunkt sowohl auf den für die zeitgenössische internationale Öffentlichkeit als auch für die Kommunismusforschung rätselhaften, erklärungsbedürftigen Phänomen der Genese des Terrors. Der facettenreiche stalinistische Terror wurde aus dem Konnex von (kommunistischer) »Ideologie und Terror« (Arendt) heraus erklärt. Hierbei wurde - zumindest was die noch zu Lebzeiten Stalins veröffentlichten Beiträge anbelangte - als interpretatorischer (Forschungs-)Ansatz ein personen-, also stalinzentriertes Modell favorisiert, das grosso modo mit einer totalitarismustheoretischen Perspektive korrespondierte. Allerdings wurde in diesem Zusammenhang deutlich, dass Stalin nach dem Tod Lenins lange Jahre keineswegs der alleinige >Herr und Meister der kommunistischen Bewegung und Partei in der Sowjetunion war und nicht von Anbeginn als Alleinverantwortlicher und -herrscher fungierte. Das heißt, bei der politischen und wissenschaftlichen Analyse des sowjetischen Kommunismus unter der Führung Stalins wurde mit Blick auf die >Frühphase deutlich, dass sich erstens der Generalsekretär der Partei insbesondere gegen seine Hauptrivalen Trotzki und Sinowjew als Nachfolger Lenins durchsetzen konnte; zweitens, dass trotz alledem in der Führungsspitze der sowjetischen Staatspartei und im Politbüro angesichts der innen- und außenpolitischen Situation zum Teil fundamentale Meinungsverschiedenheiten sowie Rivalitäts- und Richtungskämpfe vorherrschten. Folglich war es in diesem Zusammenhang 
keineswegs vorbestimmt, ob sich beispielsweise Stalin im Politbüro Ende der 1920erJahre mit der Einführung des ersten Fünfjahresplanes bzw. seines Programms der "Zweiten Revolution«, also der Doktrin vom »Aufbau des Sozialismus in einem Land«, die die Politik der Zwangskollektivierung der Landwirtschaft ins Leben rief und Millionen von Kulaken und Bauern in der Ukraine das Leben kostete, durchsetzen würde; drittens, dass auch und vor allem angesichts der katastrophalen Folgen der Politik der Zwangskollektivierung eine tatsächliche »Stalin-Opposition« von führenden Vertretern der bolschewistischen Partei um den Leningrader Parteisekretär Sergej M. Kirow existierte, sodass in Bezug auf die Analyse des Sowjetkommunismus für die späten 1920er- und frühen 1930er-Jahre festgestellt werden kann, dass die Kommunismusexperten des Monat, was diesen Zeitraum anbelangte, keineswegs von einem genuinen »Einmannregime« und einer monolithischen Herrschaftsstruktur ausgingen. In diesem Zusammenhang wurde zudem ohne Zweifel im Kontext der Beschreibung und Analyse zentraler Herrschaftsinstitutionen (unter anderem Politbüro, Generalsekretariat, staatliche Sicherheitsorgane) der Fokus sowohl auf die Entstehungsphase als auch auf die sich im Laufe der Jahre jeweils verändernde genuine Funktion gerichtet - wie beispielsweise auf das »herausragende« System der Gefängnisse und Konzentrationslager, das in der Phase des russischen Bürgerkrieges eine primär politische Funktion innehatte und letzten Endes in zeitlicher Koinzidenz mit dem einsetzenden stalinschen Industrialisierungsprogramm Ende der 1920er-Jahre neben der politischen auch und vor allem eine ökonomische Bedeutung besaß. Insofern wurde im Monat genau beleuchtet, dass mit dem Entstehen des Gulagsystems unter der Leitung des sowjetischen Geheimdienstapparates im Jahre 1930 die Zwangsarbeit in den Lagern insbesondere bei den industriellen Großbauprojekten eine zentrale Rolle spielte, die Häftlingszahlen in atemberaubenden Tempo anwuchsen und die von Millionen Menschen geleistete Zwangsarbeit zu einem elementaren Bestandteil der wirtschaftlichen Planung der sowjetischen Staatsführung avancierte.

Wie gezeigt werden konnte, wurde der »Große Terror« des sowjetischen Herrschaftsregimes insbesondere der Jahre 1936 bis 1938, der seinerzeit zum Synonym des stalinistischen Terrors wurde, ausführlich beschrieben und vor dem Hintergrund der zeitgenössischen (Forschungs-)Literatur auch mittels einer totalitarismustheoretischen Perspektive einiger Autoren (wie Borkenau oder Lasky) substanziell analysiert. So kam im Monat genau zum Ausdruck, dass nach dem Kirow-Mord am 1. Dezember 1934 auf persönliche Verantwortung bzw. direkten Befehl Stalins der Terror in Gang kam, vom NKWD-Apparat zuerst unter der Führung Jagodas und nach seiner Verhaftung ab 1936 von Jeschow durchgeführt wurde und seinen mörderischen »Höhepunkt « einerseits in den drei öffentlichen Schauprozessen, der permanenten Säuberung der Partei sowie der staatlichen Apparate (Sicherheitsorgane und Armee), also der "großen Tschistka«, und andererseits in den Massenverbrechen gegen die sowjetische Bevölkerung hatte. Kurzum: Der permanente Terror des stalinistischen Regimes der Jahre 1936 bis 1938 wurde im Monat ausführlich thematisiert und beschrieben und es wurde der Versuch gemacht, ihn zu interpretieren. In diesem Kontext stellte der hier ausführlich vorgestellte Beitrag Die Moskauer Geständnisse. Gedanken über Ketzerei und Opposition im totalen Staat, d. h. die Rezension der grundlegenden Studie Russian Purge and the Extraction von Beck und Godin aus dem Jahre 1951, von Lasky die substanziellste Analyse dar. Hier kam in nuce zum Ausdruck, wie der stalinistische Terror funktionierte: In dem nämlich vor dem Hintergrund des totalitären Führers und der zentra- 
len Entscheidungsinstanz des sowjetischen Staates, also Stalin, und der - keineswegs per se rätselhaften - öffentlichen Geständnisse der Angeklagten der Schauprozesse, d.h. der prominenten führenden Altbolschewiken wie Bucharin, Rykow oder Jagoda, durch die Interdependenz der »Apparate«, der Folterpraxis, der ideologischen Feindbilder, der irrealen Verschwörungstheorien und der sogenannten Kontaktschuld eine selbstreferenzielle bürokratische Verfolgungslogik in Gang kam, sodass schlussendlich der Terror für diese zwei Jahre eine »dauernde Institution« (Borkenau) wurde. Zudem konnte gezeigt werden, dass in diesem Kontext der analytische Fokus - idealtypisch gesprochen - auch auf sämtliche staatlichen Institutionen und die sowjetische Gesellschaft in toto gerichtet wurde. Denn wie aus den Ausführungen und Reflexionen Laskys deutlich wurde: Der stalinistische Terror ließ sich nicht ausschließlich durch die totalitäre politische Macht und den "pathologischen Verfolgungswahn" Stalins sowie die genuine Verfolgungs- und Verhaftungspraxis des NKWD-Apparates reduzieren, geschweige denn angemessen historisch interpretieren bzw. erklären. Vor dem Hintergrund der in Gang gekommenen Parteisäuberung und des politischen Terrors gegen die Bevölkerung entstand nämlich tatsächlich in der Sowjetunion ein gesellschaftliches Klima, in dem jeder Bürger verdächtigt und zum »Feind« erklärt werden konnte und gewissermaßen der gesamte Alltag des Sowjetbürgers von einer »Feindobsession« bzw. "Feindparanoia« infiziert war, sodass schließlich die von Arendt beschriebene und analysierte »Atomisierung « entstand, d.h. jene »Massengesellschaft terrorisierter und isolierter Individuen«, die wiederum die gegenseitige Denunziation der einzelnen Parteimitglieder der KPdSU (einschließlich der Politemigranten der Komintern, der KPD und weiterer Funktionäre einzelner KPs) und der Menschen untereinander - sogar bis in die eigene Familie - sowie bei den sowjetischen Sicherheitsorganen zur Folge hatte. Hierdurch wurde der stalinistische Terror nicht nur weiter in Gang gehalten, sondern radikalisierte sich mittels der selbstreferenziellen bürokratischen Verfolgungslogik der Sicherheitsorgane zusätzlich.

Vor diesem Hintergrund kann festgestellt werden, dass der stalinistische Terror der 1930er-Jahre und der Jahre 1936 bis 1938 im Besonderen im Monat konkret, d.h. empirisch ausgeleuchtet und untersucht wurde. Zum Beispiel wurde der NKWD-Terror und speziell die genuine brutale Verhör- und Folterpraxis der stalinistischen Täter von Weißberg-Cybulski in seinem Beitrag Das große Verhör. Aus der Praxis des NKWD (H.35) beschrieben - hierbei handelte es sich um einen Auszug aus seinem 1951 erschienenen autobiografischen (Renegaten-)Buch Hexensabbath. Rußland im Schmelztiegel der Säuberungen -, auf den sich auch Lasky in Die Moskauer Geständnisse. Gedanken über Ketzerei und Opposition im totalen Staat (H.36) stützte. In diesem Zusammenhang kann festgestellt werden, dass auch die stalinistischen Massenverbrechen an der sowjetischen Bevölkerung in den Blick genommen wurden - nicht zuletzt im Kontext der vielschichtigen Thematisierung des Systems der Konzentrationslager und des stalinschen Programms der Zwangskollektivierung Ende 1920/Anfang 1930 mit den Millionen von Toten insbesondere unter den ukrainischen »Kulaken«. Allerdings wurden die konkreten Massenverbrechen und der konkrete Terror an der sowjetischen Bevölkerung nicht ausdrücklich beschrieben und analysiert. Das lag zweifelsohne einerseits an der schon mehrmals zur Sprache gebrachten Situation von fehlenden zuverlässigen und elementaren Dokumenten und Statistiken (kurz: Archivsituation), mit der die zeitgenössische Sowjetforschung grundsätzlich konfrontiert war. Andererseits lag dies - so die These - an einer Elitenperspektive zahlreicher Autoren im Monat, 
wie beispielsweise bei Borkenau, Löwenthal oder auch zum Teil bei Koestler, die als ehemalige führende Funktionäre der Kommunistischen Partei den Schwerpunkt in erster Linie auf die »Führer« wie Lenin, Stalin und Chruschtschow sowie die zentralen Institutionen wie die Parteispitze, das Politbüro, den Geheimdienstapparat oder die Komintern richteten.

Ebenso wie zum Beispiel der überaus komplexe stalinistische Terror in den 1930erJahren wurde die zeitgenössische Entwicklung des kommunistischen Herrschaftssystems und speziell des sowjetischen Regimes nach dem Ende des Zweiten Weltkrieges bis zum Tod Stalins am 5. März 1953 und des Nachstalinismus in zahlreichen Beiträgen thematisiert und beschrieben sowie der Versuch gemacht, den alsbald wieder beginnenden (Massen-)Terror - unter anderem die Verhaftungen und Deportationen von Millionen Menschen in die Konzentrationslager, die einzelnen Schauprozesse und Säuberungswellen in der KPdSU und den einzelnen KPs vor allem in den osteuropäischen Satellitenstaaten - zu interpretieren. In diesem Zusammenhang avancierte der international bekannte deutsche Emigrant und Renegat Richard Löwenthal zum eigentlichen Experten der Zeitschrift, der - abgesehen von dem Schweizer Historiker und Frankreichexperten Herbert Lüthy - nicht nur die zahlenmäßig meisten Beiträge zu ebendiesem Themenkomplex veröffentlichte, sondern zudem die substanziellsten Analysen erstellte. Hierbei konnte unter anderem gezeigt werden, dass der Schwerpunkt der löwenthalschen Analysen auf der gewissermaßen totalitären Triebkraft des Stalinismus (und im Nachstalinismus) lag, sprich: dem »Gesetz der permanenten Revolution«bzw. der »permanenten Revolution von oben«, die mit einer totalitarismustheoretischen Perspektive korrespondierten. Insofern wurde der analytische Fokus auf die zentralen Herrschaftsinstitutionen gerichtet, d. h. in erster Linie auf die von Stalin beherrschte Partei, das Politbüro, den Geheimdienstapparat oder auch auf das Generalsekretariat. Gleichwohl wurde in diesem Zusammenhang in dem noch zu Lebzeiten des unumstrittenen Staats- und Parteiführers Stalin verfassten Beitrag Der Hintergrund der Säuberung (H. 54) von Löwenthal festgestellt, dass es innerhalb des Politbüros essenzielle Meinungsverschiedenheiten in Bezug auf den richtigen innen- und außenpolitischen Kurs der imperialistischen Sowjetunion gab - in erster Linie angesichts der defensiven Position Moskaus, d. h. der gebremsten außenpolitischen Expansion in der zugespitzten Situation angesichts des ausgebrochenen Kalten Krieges (Berlinkrise etc.). Darüber hinaus wurde auch auf die politisch-ideologischen Machtkämpfe in der sowjetischen Führung zu Zeiten Stalins unter seinen zukünftigen potenziellen Nachfolgern eingegangen. Folglich wurden auch zeitgenössische Vorstellungen implizit infrage gestellt, wonach in dem totalitären Regime alle führenden Funktionäre der herrschenden Staatspartei ausschließlich nach der »Pfeife« Stalins tanzen sowie aus Angst keine unabhängigen Positionen vertreten und keine eigenständigen politische Ziele verfolgen würden.

Mehr noch: In den nach dem Tod Stalins verfassten Beiträgen Löwenthals - die an dieser Stelle stellvertretend für zahlreiche weitere Artikel im Monat stehen und die sich in erster Linie mit der weiteren tatsächlichen und potenziellen Entwicklung des sowjetischen Regimes auseinandersetzten - wurde bei der Analyse des Nachstalinismus ein genuin nichtpersonenzentrierter, d.h. polykratischer (Forschungs-) Ansatz favorisiert. Bemerkenswerterweise wurde in diesem Kontext post festum auch ausgehend von der »Vier-Säulentheorie« (Parteiapparat, Geheimpolizei, Armee sowie staatliche Wirtschaftsverwaltung) bereits für den Stalinismus eine nicht vorhandene 
"monolithische Staatsstruktur" (Arendt) und dementsprechend eine "Strukturlosigkeit« des totalitären Herrschaftsregimes beschrieben und analysiert. Es wurde also sehr wohl davon ausgegangen, dass es sich bei den unterschiedlich ausgeprägten sowjetischen Herrschaftsregimen unter Stalin (Stalinismus) und nach seinem Tod unter der »kollektiven Führung « sowie Mitte und dann Ende der 1950er-Jahre unter dem »neuen Führer« Chruschtschow (Nachstalinismus) gleichwohl um den Typus eines Herrschaftsregimes handelte, das grundsätzlich durch die Tatsache der »doppelten Autorität von Staat und Partei« (Arendt) charakterisiert war. Daraus folgt auch, dass im Monat in diesem Kontext ein polykratischer (Forschungs-)Ansatz vertreten wurde - Löwenthal steht hier pars pro toto.

Die Genesis des nachstalinistischen Herrschaftssystems einschließlich der osteuropäischen Satellitenstaaten sowie der einzelnen kommunistischen Parteien in den westeuropäischen Ländern (vor allem in Italien und Frankreich) wurde ausführlich thematisiert, beschrieben und analysiert. In diesem Zusammenhang kam nach dem Tod Stalins unter den Autoren und renommierten internationalen Kommunismusexperten des Monat ein Diskussionsprozess in Gang, in dessen Mittelpunkt aufgrund der facettenreichen fundamentalen politischen und gesellschaftlichen Veränderungen im sowjetkommunistischen Herrschaftssystems die genuin analytisch ausgerichtete Frage nach der zeitgenössischen Natur des Regimes stand. Diese Entwicklung fand nach dem »langen Weg « Chruschtschows - einerseits nach Machtkämpfen in der Führung und zahlreichen »blutigen « und vor allem »unblutigen« Säuberungen nicht zuletzt in der KPdSU und den sowjetischen staatlichen Sicherheitsorganen, andererseits nach den krisenhaften Entwicklungen in den osteuropäischen Satellitenstaaten 1956 - seinen vorläufigen »Höhepunkt « in seiner Alleinherrschaft im Jahre 1959. Hierbei wurde angesichts der Untersuchung der ökonomischen bzw. industriellen Entwicklung des Landes und der personellen, institutionellen und ideologischen Veränderungen sowie nicht zuletzt der `Zähmung der Geheimpolizei im sowjetkommunistischen Einparteienregime, also nach der Zerschlagung des "Stalinschen Super-Apparats« (Löwenthal), auch die Bedeutung des Nachlassens des Terrors und die damit verbundene »Liberalisierung« ins Zentrum gerückt. Insbesondere im Kontext des XX. Parteitages der KPdSU sowie der chruschtschowschen Geheimrede im Februar 1956 wurde insbesondere die für die internationale Öffentlichkeit und Wissenschaft gewissermaßen auf den »Nägeln« brennende Frage auch unter den Experten des Monat durchaus kontrovers diskutiert, ob es sich bei dem »neuen« Herrschaftsregime unter Chruschtschow noch um eine totalitäre Diktatur handeln würde und inwiefern mithin der Begriff des Totalitarismus zur Charakterisierung des sowjetischen Systems noch angemessen wäre (exemplarisch sei an dieser Stelle die oben ausführlich referierte, in der Juniausgabe des Jahres 1956 veröffentlichte Umfrage zu dem Themenkomplex »Ist Rußland anders geworden?« genannt, an der Silone, Macdonald, Crankshaw, Halperin, Lippmann und Borkenau teilnahmen).

Für den hier zugrunde liegenden Untersuchungszeitraum (Oktober 1948 bis April 1960) kann für den Monat festgestellt werden, dass im Hinblick auf die Analyse des sowjetischen Kommunismus von Anfang an erstens die wichtigsten Studien der zeitgenössischen internationalen Forschungsliteratur zur Geschichte des Sowjetkommunismus entweder in Form einer Buchrezension rezipiert oder sogar als Vor- bzw. Teilabdruck veröffentlicht wurden, zweitens zahlreiche international renommierte Kommunismusexperten als Mitarbeiter gewonnen worden waren, drittens, dass das 
theoretische und historische Wissen nicht nur der Renegaten, sondern zahlreicher der im Kapitel II.4 dieser Arbeit porträtierten »Exponenten des Monat« (vor allem Borkenau, Löwenthal, Koestler, Bondy, Lüthy, Kohn oder auch Aron) genutzt wurde. Überhaupt kann im Hinblick auf die Auseinandersetzung mit der Genesis des facettenreichen Phänomens des sowjetkommunistischen Herrschaftssystems festgestellt werden, dass es der Zeitschrift Der Monat um tatsächliche und grundsätzliche politisch-wissenschaftliche Aufklärung ging.

Die im Monat veröffentlichten Beiträge zu dem Themenkomplex Analyse des Sowjetkommunismus deckten sich, sowohl was die inhaltliche Ausrichtung als auch was die historisch-politische Auseinandersetzung mit der Geschichte des facettenreichen Phänomens des internationalen Kommunismus anbelangte, mit der in diesen Jahren führenden wissenschaftlichen Zeitschrift in der Bundesrepublik, d.h. dem "Informationsblatt« (Wolfgang Leonhard) Ost-Probleme. Wie eingangs bereits angesprochen, veröffentlichte hierbei nicht nur in zahlreichen Fällen derselbe Personenkreis von internationalen Kommunismusexperten in den beiden Organen, sodass sich naheliegenderweise die einzelnen Kommentare, Interpretationen und Analysen deckten, sondern darüber hinaus wurde im Monat und in Ost-Probleme größtenteils auch die gleiche Forschungsliteratur rezipiert und besprochen. Insofern kann es post festum nicht verwundern, dass beispielsweise in der 1959 erschienenen wichtigen Studie Kreml ohne Stalin von Leonhard als Quelle auch und vor allem zahlreiche zentrale Beiträge von ebenjenen international renommierten Kommunismusexperten dienten, die im Monat und in Ost-Probleme veröffentlicht wurden.

\section{Vergleichende Analyse der totalitären Herrschaftssysteme des Nationalsozialismus und des Sowjetkommunismus}

\subsection{Vorbemerkung und Fragestellung}

Nachdem in den beiden vorherigen Kapiteln die Beschreibung und Analyse der Genese und Entwicklung des Nationalsozialismus sowie des Sowjetkommunismus im Monat aufder Basis des jeweils entwickelten Fragenkomplexes untersucht wurde - im Fokus stand hierbei die Frage, welches Bild die Zeitschrift jeweils in ihren Beiträgen in den Jahren von Ende 1948 bis Mitte 1960 von den beiden Herrschaftssystemen zeichnete, welche Autoren hierzu veröffentlichten und letztlich zu den Experten avancierten -, soll es im Folgenden mit Blick auf die zeitgenössischen wissenschaftlichen Totalitarismusstudien um den ausdrücklichen Vergleich zwischen den beiden totalitären Herrschaftssystemen des 20. Jahrhunderts, also des deutschen Nationalsozialismus und des Sowjetkommunismus, gehen. ${ }^{599}$ Vor dem Hintergrund des auch und vor allem in der Totalitarismusforschung einflussreichen Konzeptes der "politischen Religionen«

599 Im Gegensatz zu den beiden vorherigen Kapiteln ist in diesem Kapitel die Frage, welche Autoren zum Thema vergleichende Analyse der totalitären Herrschaftssysteme des Nationalsozialismus und des Sowjetkommunismus veröffentlichten und zu den Totalitarismus-Experten avancierten, nicht von Interesse. Nur soviel: Es hat sich letzten Endes hier kein Autor des Monat zum eigentlichen Experten herauskristallisiert. Strenggenommen wäre dies Orwell, von dem fünf Beiträge zu diesem Themenkomplex erschienen, und zwar im Kontext der Veröffentlichung seines Romanes 1984. 